\title{
DISTANCE SPECTRAL RADIUS OF SERIES-REDUCED TREES WITH PARAMETERS
}

\author{
Yuyuan Deng ${ }^{1}$, DAngui Li ${ }^{1}$, Hongying Lin ${ }^{2}$ And Bo Zhou ${ }^{1, *}$
}

\begin{abstract}
For a connected graph $G$, the distance matrix is a real-symmetric matrix where the $(u, v)$ entry is the distance between vertex $u$ and vertex $v$ in $G$. The distance spectral radius of $G$ is the largest eigenvalue of the distance matrix of $G$. A series-reduced tree is a tree with at least one internal vertex and all internal vertices having degree at least three. Those series-reduced trees that maximize the distance spectral radius are determined over all series-reduced trees with fixed order and maximum degree and over all series-reduced trees with fixed order and number of leaves, respectively.
\end{abstract}

Mathematics Subject Classification. 05C50, 05C35, 15A48.

Received April 17, 2020. Accepted August 30, 2020.

\section{INTRODUCTION}

All graphs in this paper are finite, simple and undirected. Let $G$ be a graph of order $n$ with vertex set $V(G)$ and edge set $E(G)$. For $u, v \in V(G)$, the distance between $u$ and $v$ in $G$, denoted by $d_{G}(u, v)$, is the length of a shortest path joining $u$ and $v$ in $G$. The distance matrix of $G$ is the $n \times n$ matrix $D(G)=\left(d_{G}(u, v)\right)_{u, v \in V(G)}$. It is a symmetric matrix, and therefore, all its eigenvalues are real. The largest eigenvalue of $D(G)$ is called the distance spectral radius of $G$, denoted by $\rho(G)$.

\subsection{Problems and existing results}

A natural question is what can be said about the distance spectral radius. Let $\mathbb{G}$ be a class of connected graphs. It is natural and of interest to find

$$
\min \{\rho(G): G \in \mathbb{G}\}
$$

and

$$
\max \{\rho(G): G \in \mathbb{G}\}
$$

and determine the graphs in $\mathbb{G}$ attaining the minimum and maximum. One of the first results on the spectrum of the distance matrix of a graph was obtained by Graham and Pollak [8], who showed that if $G$ is a tree on $n$ vertices, then $\operatorname{det}(D(G))=(-1)^{n-1}(n-1) 2^{n-2}$, and when $n \geq 2, D(G)$ has one positive eigenvalue and $n-1$

Keywords. Distance spectral radius, distance matrix, maximum degree, number of leaves, series-reduced tree.

1 School of Mathematical Sciences, South China Normal University, Guangzhou 510631, P.R. China.

2 School of Mathematics, South China University of Technology, Guangzhou 510641, P.R. China.

*Corresponding author: zhoubo@scnu.edu.cn 
negative eigenvalues. This unexpected result also aroused the study of the spectrum of the distance matrix to explore the roles of separate eigenvalues, especially the role of the distance spectral radius. In applications, the distance spectral radius has been successfully used as a molecular descriptor [2,9]. Zhou and Trinajstić [29] provided lower and upper bounds for the distance spectral radius in terms of the order, the sum of squares of the distances between all unordered pairs of vertices, and the status (sums of the distances between a vertex and all other vertices). Ruzieh and Powers [23] initiated in 1990 the study to determine the graphs that maximize or minimize the distance spectral radius in a class of graphs, and they showed that the maximum possible value for all connected graphs on $n$ vertices is achieved by the path, for which the distance spectral radius is $\frac{n^{2}}{2 a^{2}}-\frac{2+a^{2}}{6 a^{2}}+O\left(n^{-2}\right)$, where $a$ is the root of $a \tanh (a)=1$. Stevanović and Ilić [24] showed that the broom consisting of $\Delta-1$ edges and a path of length $n-\Delta$ at a common vertex maximizes uniquely the distance spectral radius over all trees of order $n$ with maximum degree $\Delta \geq 2$. In recent ten years, there are lots of papers on the distance spectral radius, and among them, lots of work was devoted to the distance spectral radius of trees. There is a survey of Aouchiche and Hansen [1] in 2014 on the relevant background and results on the spectrum of the distance matrix of a graph, and especially on the distance spectral radius. The extremal results on the distance spectral radius of trees may be found in $[12,20]$ with fixed matching number, in $[20,21]$ with given number of leaves, in $[15,17,18,24]$ with given maximum degree, in $[6,28]$ with fixed diameter, in [14] with fixed number of odd vertices, in [25] with fixed domination number, in [16] with given number of vertices of degree two, in [5] with fixed degree sequence, and in $[15,16,26,27]$ for some particular classes of trees.

\subsection{Work of this paper}

A vertex of degree one in a graph is called a leaf. A vertex of a tree is an internal vertex if it is not a leaf. A series-reduced tree is a tree with at least one internal vertex and all internal vertices having degree at least three. It is also known as a homeomorphically irreducible tree [11]. Series-reduced trees form an important family of trees $[3,7,10,11]$. So it is natural to ask what can be said about the connection between the extremal distance spectral radius and structural parameters of series-reduced trees, which is the goal of this article. As examples, two parameters including maximum degree and number of leaves are considered. Those trees that maximize the distance spectral radius are determined over all series-reduced trees of order $n$ with maximum degree $\Delta$, where $3 \leq \Delta \leq n-5$, and over all series-reduced trees of order $n$ with $p$ leaves, where $\left\lceil\frac{n+2}{2}\right\rceil \leq p \leq n-2$, respectively.

\section{Preliminaries}

For a graph $G$ with $u \in V(G)$, let $N_{G}(u)=\{v \in V(G): u v \in E(G)\}$. The degree of vertex $u$ in $G$ is $\delta_{G}(u)=\left|N_{G}(u)\right|$.

A diameter of a connected graph $G$ is the greatest distance between vertices of $G$.

An edge which is incident with a leaf is called a leaf edge. For a tree $T$ with a leaf $v, v$ is said to be a leaf at $u$ if $v$ is adjacent to $u$ in $T$. Given a nontrivial tree $T$ with $u \in V(T)$, if a new vertex $v$ and a new edge $u v$ are added to $T$ to form a new tree $T^{\prime}$, then $T^{\prime}$ is said to be obtained from $T$ by adding a leaf edge $u v$ or a leaf $v$ at $u$.

The status of a vertex $u$ of a connected graph $G$ is $s_{G}(u)=\sum_{v \in V(G)} d_{G}(u, v)$, and the minimum status of $G$ is $s(G)=\min \left\{s_{G}(u): u \in V(G)\right\}$. Evidently, $s(G)$ equals the minimum row sum of $D(G)$. It is easy to see that the minimum status of a tree of order at least three is achieved by some internal vertex, see, e.g., [22].

Let $G$ be a connected graph with $V(G)=\left\{v_{1}, \ldots, v_{n}\right\}$ and $x=\left(x_{v_{1}}, \ldots, x_{v_{n}}\right)^{\top}$ a real column vector. Then

$$
x^{\top} D(G) x=\sum_{\{u, v\} \subseteq V(G)} 2 d_{G}(u, v) x_{u} x_{v} .
$$

As $D(G)$ is a nonnegative irreducible matrix, by the well known Perron-Frobenius theorem, $\rho(G)$ is simple, and associating with $\rho(G)$, there is a unique positive unit eigenvector, which is called the $D(G)$-Perron vector. 


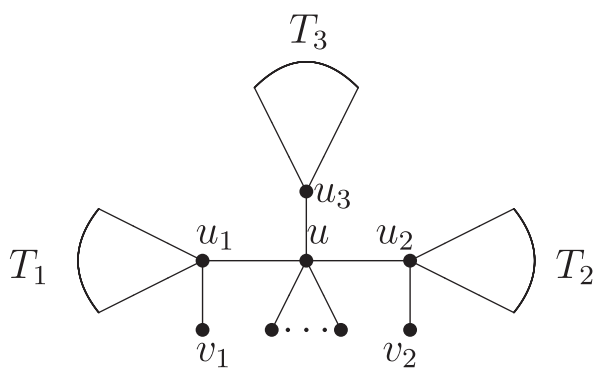

FIgURE 1. The tree $T$ in Lemma 2.4.

If $x$ is the $D(G)$-Perron vector, then, for each $u \in V(G)$,

$$
\rho(G) x_{u}=\sum_{v \in V(G)} d_{G}(u, v) x_{v} .
$$

This equation is called the $D(G)$-eigenequation at $u$. By Rayleigh's principle, for a unit nonnegative column vector $y=\left(y_{v_{1}}, \ldots, y_{v_{n}}\right)^{\top}$,

$$
\rho(G) \geq y^{\top} D(G) y
$$

with equality if and only if $y$ is the $D(G)$-Perron vector.

If $E_{1}$ is a set of unordered pairs of distinct vertices that are not adjacent in $G$, then $G+E_{1}=(V(G)$, $\left.E(G) \cup E_{1}\right)$, and if $E_{1} \subseteq E(G)$, then $G-E_{1}=\left(V(G), E(G) \backslash E_{1}\right)$. If $E_{1}=\{u v\}$, then $G+u v$ or $G-u v$ is used for $G+E_{1}$ or $G-E_{1}$. If $u \in V(G)$, then $G-u$ is the subgraph of $G$ obtained by deleting $u$ and all edges incident with $u$.

For a subset $V_{0}$ of vertices of a connected graph $G, \sigma_{G}\left(V_{0}\right)$ denotes the sum of the entries of the $D(G)$-Perron vector corresponding to the vertices from $V_{0}$. If $H$ is an induced subgraph of $G, \sigma_{G}(H)$ is used for $\sigma_{G}(V(H))$.

Lemma $2.1([25])$. Let $G$ be a connected graph with $u, v \in V(G)$ and $x$ the $D(G)$-Perron vector. Suppose that $u^{\prime}$ and $v^{\prime}$ are leaves adjacent to $u$ and $v$, respectively. Then $x_{u}^{\prime}-x_{v}^{\prime}=\frac{\rho(G)}{\rho(G)+2}\left(x_{u}-x_{v}\right)$.

Note that in previous lemma, $u$ and $v$ are not necessarily distinct.

Lemma 2.2 ([25]). Let $G$ be a connected graph and uv a cut edge but not a leaf edge of $G$. Let $G^{\prime}=G-\{v w$ : $\left.w \in N_{G-u v}(v)\right\}+\left\{u w: w \in N_{G-u v}(v)\right\}$. Then $\rho\left(G^{\prime}\right)<\rho(G)$.

Lemma 2.3 ([14]). Let $T$ be a tree with $u \in V(T)$, and let $N_{T}(u)=\left\{u_{1}, \ldots, u_{k}\right\}$, where $k \geq 3$. Let $T_{i}$ be the component of $T-u$ containing $u_{i}$ for $1 \leq i \leq k$. Let $T^{\prime}=T-\left\{u u_{i}: 3 \leq i \leq t\right\}+\left\{w u_{i}: 3 \leq i \leq t\right\}$, where $3 \leq t \leq k$ and $w \in V\left(T_{2}\right)$. If $\sigma_{T}\left(T_{1}\right) \geq \sigma_{T}\left(T_{2}\right)$, then $\rho\left(T^{\prime}\right)>\rho(T)$.

Lemma 2.4. Let $T$ be a series-reduced tree on $n$ vertices whose structure is displayed in Figure 1, where $u_{1}, u_{2}$, and $u_{3}$ are neighbors of $u$, each neighbor of $u$ different from $u_{1}, u_{2}, u_{3}$ (if any exists) is a leaf in $T, v_{i}$ is a leaf at $u_{i}$ for $i=1,2$, and $\delta_{T}\left(u_{3}\right) \geq 3$. Let $T^{\prime}=T-\left\{u u_{3}, u_{1} v_{1}\right\}+\left\{u_{1} u_{3}, u v_{1}\right\}$ and $T^{*}=T-\left\{u u_{3}, u_{2} v_{2}\right\}+\left\{u_{2} u_{3}, u v_{2}\right\}$. Then $\rho\left(T^{\prime}\right)>\rho(T)$ or $\rho\left(T^{*}\right)>\rho(T)$.

Proof. Because $T$ is a series-reduced tree, $T^{\prime}$ is also a series-reduced tree. Let $x$ be the $D(T)$-Perron vector. Let $N_{u}=N_{T}(u) \backslash\left\{u_{1}, u_{2}, u_{3}\right\}$. Let $T_{1}, T_{2}$ and $T_{3}$ be the three nontrivial components in $T-u$ containing $u_{1}, u_{2}$ and $u_{3}$, respectively.

Suppose that $\sigma_{T}\left(T_{1}\right) \leq \sigma_{T}\left(T_{2}\right)$. As $T$ is transformed into $T^{\prime}$, the distance between a vertex of $V\left(T_{3}\right)$ and a vertex of $V\left(T_{2}\right) \cup\{u\} \cup N_{u}$ is increased by 1 , the distance between a vertex of $V\left(T_{3}\right)$ and a vertex of $V\left(T_{1}\right) \backslash\left\{v_{1}\right\}$ 


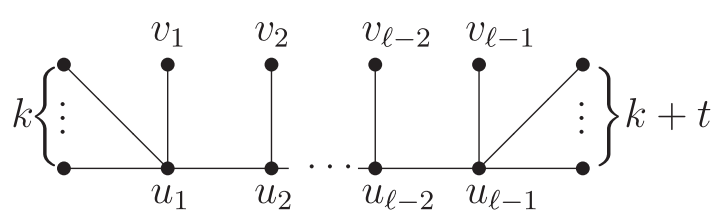

Figure 2. The tree $E(n, \ell ; k, k+t)$.

is decreased by 1 , the distance between $v_{1}$ and a vertex of $V\left(T_{1}\right) \backslash\left\{v_{1}\right\}$ is increased by 1 , the distance between $v_{1}$ and a vertex of $V\left(T_{2}\right) \cup\{u\} \cup N_{u}$ is decreased by 1, and the distances between all other pairs of vertices remain unchanged. Thus

$$
\begin{aligned}
\frac{1}{2}\left(\rho\left(T^{\prime}\right)-\rho(T)\right) \geq & \frac{1}{2} x^{\top}\left(D\left(T^{\prime}\right)-D(T)\right) x \\
= & \sigma_{T}\left(T_{3}\right)\left(\sigma_{T}\left(T_{2}\right)+x_{u}+\sigma_{T}\left(N_{u}\right)-\left(\sigma_{T}\left(T_{1}\right)-x_{v_{1}}\right)\right) \\
& +x_{v_{1}}\left(\sigma_{T}\left(T_{1}\right)-x_{v_{1}}-\left(\sigma_{T}\left(T_{2}\right)+x_{u}+\sigma_{T}\left(N_{u}\right)\right)\right) \\
= & \left(\sigma_{T}\left(T_{2}\right)-\sigma_{T}\left(T_{1}\right)+x_{u}+\sigma_{T}\left(N_{u}\right)+x_{v_{1}}\right)\left(\sigma_{T}\left(T_{3}\right)-x_{v_{1}}\right) .
\end{aligned}
$$

Let $a, b$ be two neighbors of $u_{3}$ in $T_{3}$. For any $v \in V(T) \backslash\left\{u_{3}, a, b, v_{1}\right\}, d_{T}\left(u_{3}, v\right)+d_{T}(a, v)+d_{T}(b, v)-d_{T}\left(v_{1}, v\right) \geq 0$. From the $D(T)$-eigenequations at $u_{3}, a, b$ and $v_{1}$, one has

$$
\begin{aligned}
\rho(T)\left(x_{u_{3}}+x_{a}+x_{b}-x_{v_{1}}\right)= & \sum_{v \in V(T)}\left(d_{T}\left(u_{3}, v\right)+d_{T}(a, v)+d_{T}(b, v)-d_{T}\left(v_{1}, v\right)\right) x_{v} \\
= & \sum_{v \in V(T) \backslash\left\{u_{3}, a, b, v_{1}\right\}}\left(d_{T}\left(u_{3}, v\right)+d_{T}(a, v)+d_{T}(b, v)-d_{T}\left(v_{1}, v\right)\right) x_{v} \\
& +(0+1+1-3) x_{u_{3}}+(1+0+2-4) x_{a} \\
& +(1+2+0-4) x_{b}+(3+4+4-0) x_{v_{1}} \\
\geq & -x_{u_{3}}-x_{a}-x_{b}+11 x_{v_{1}}
\end{aligned}
$$

and thus $(\rho(T)+1)\left(x_{u_{3}}+x_{a}+x_{b}-x_{v_{1}}\right) \geq 10 x_{v_{1}}>0$, which implies $x_{u_{3}}+x_{a}+x_{b}-x_{v_{1}}>0$. Therefore, $\sigma_{T}\left(T_{3}\right)-x_{v_{1}} \geq x_{u_{3}}+x_{a}+x_{b}-x_{v_{1}}>0$. Evidently, $\sigma_{T}\left(T_{2}\right)-\sigma_{T}\left(T_{1}\right)+x_{u}+\sigma_{T}\left(N_{u}\right)+x_{v_{1}} \geq x_{u}+\sigma_{T}\left(N_{u}\right)+x_{v_{1}}>0$. From (2.1), one has $\rho\left(T^{\prime}\right)>\rho(T)$.

If $\sigma_{T}\left(T_{2}\right)<\sigma_{T}\left(T_{1}\right)$, then by similar arguments as above, one has $\rho\left(T^{*}\right)>\rho(T)$.

Recall that a caterpillar is a tree in which removal of all leaves outside a longest path (if any exists) gives a path.

For simplicity, $[n]$ is used for $\{1, \ldots, n\}$ if $n$ is a positive integer.

For integers $n, \ell, k, t$ with $n=2 \ell+2 k+t-2, \ell \geq 2, k \geq 0$ and $t \geq 0$, let $E(n, \ell ; k, k+t)$ be a caterpillar obtained from $P_{\ell-1}$ whose vertices are consecutively labelled as $u_{1}, \ldots, u_{\ell-1}$ by adding a leaf $v_{i}$ at $u_{i}$ for $i \in[\ell-1]$ and then adding $k$ leaves at $u_{1}$ and $k+t$ leaves at $u_{\ell-1}$, see Figure 2 . Note that this tree has diameter $\ell$.

Lemma 2.5. Let $G=E(n, \ell ; k, k+t)$, where $k \geq 0, t \geq 1, \ell=\frac{n+2-2 k-t}{2}=2 d$, and $d \geq 2$. Let $x$ be the $D(G)$-Perron vector and $s_{i}=x_{u_{i}}+x_{v_{i}}$ for $i \in[2 d-1]$. Then $s_{d-i}>s_{d+i}$ for $i \in[d-1]$.

Proof. Let the vertices of $G$ be labelled as in Figure $2\left(\right.$ with $\ell=2 d$ ). Let $\Lambda=\sum_{j=d+1}^{2 d-1} s_{j}+(k+t) x_{v_{2 d-1}}-$ $\left(\sum_{j=1}^{d-1} s_{j}+k x_{v_{1}}\right)$. 
Let $K=\rho(G)+\frac{\rho(G)}{\rho(G)+1}$. Suppose that $i \in[d-1]$. By Lemma 2.1, one has

$$
\begin{aligned}
\rho(G)\left(s_{d-i}-s_{d+i}\right) & =\rho(G)\left(x_{u_{d-i}}-x_{u_{d+i}}\right)+\rho(G)\left(x_{v_{d-i}}-x_{v_{d+i}}\right) \\
& =\rho(G)\left(x_{u_{d-i}}-x_{u_{d+i}}\right)+\frac{\rho^{2}(G)}{\rho(G)+2}\left(x_{u_{d-i}}-x_{u_{d+i}}\right) \\
& =\frac{2 \rho(G)(\rho(G)+1)}{\rho(G)+2}\left(x_{u_{d-i}}-x_{u_{d+i}}\right),
\end{aligned}
$$

i.e.,

$$
K\left(s_{d-i}-s_{d+i}\right)=2 \rho(G)\left(x_{u_{d-i}}-x_{u_{d+i}}\right) .
$$

So, by the $D(G)$-eigenequations at $u_{d-i}$ and $u_{d+i}$, one has

$$
\begin{aligned}
K\left(s_{d-i}-s_{d+i}\right)= & 2\left(-2 i \sum_{j=1}^{d-i} s_{j}-2 i k x_{v_{1}}-\sum_{j=1}^{i-1} 2 j s_{d-j}\right. \\
& \left.+\sum_{j=1}^{i-1} 2 j s_{d+j}+2 i \sum_{j=d+i}^{2 d-1} s_{j}+2 i(k+t) x_{v_{2 d-1}}\right) \\
= & -4 i \sum_{j=1}^{d-i} s_{j}-4 i k x_{v_{1}}-\sum_{j=1}^{i-1} 4 j s_{d-j}+\sum_{j=1}^{i-1} 4 j s_{d+j}+4 i \sum_{j=d+i}^{2 d-1} s_{j} \\
& +4 i(k+t) x_{v_{2 d-1}} .
\end{aligned}
$$

Thus

$$
\begin{aligned}
K\left(\left(s_{d-i}-s_{d+i}\right)-\left(s_{d-(i-1)}-s_{d+(i-1)}\right)\right) & =4\left(\sum_{j=d+i}^{2 d-1} s_{j}+(k+t) x_{v_{2 d-1}}-k x_{v_{1}}-\sum_{j=1}^{d-i} s_{j}\right) \\
& =4 \Lambda+4 \sum_{j=1}^{i-1}\left(s_{d-j}-s_{d+j}\right) .
\end{aligned}
$$

It is easy to prove that $s_{d-i}-s_{d+i}$ and $\Lambda$ have the same sign by induction on $i \in[d-1]$. If $i=1$, then this follows from (2.2). Suppose that $2 \leq i \leq d-1$. By the induction hypothesis, $s_{d-j}-s_{d+j}$ and $\Lambda$ have the same sign for $j \in[i-1]$. Thus $4 \sum_{j=1}^{i-1}\left(s_{d-j}-s_{d+j}\right)$ and $\Lambda$ have the same sign. Now from $(2.2), s_{d-i}-s_{d+i}$ and $\Lambda$ have the same sign. This shows that $s_{d-i}-s_{d+i}$ and $\Lambda$ have the same sign for all $i \in[d-1]$, as desired.

Suppose that $x_{u_{1}} \leq x_{u_{2 d-1}}$. By Lemma 2.1,

$$
(2 \rho(G)+2)\left(x_{u_{1}}-x_{u_{2 d-1}}\right)=(\rho(G)+2)\left(s_{1}-s_{2 d-1}\right) .
$$

Thus $s_{1} \leq s_{2 d-1}$. As $s_{d-i}-s_{d+i}$ and $\Lambda$ have the same sign for $i \in[d-1], \Lambda+\sum_{j=1}^{d-1}\left(s_{d-j}-s_{d+j}\right) \leq 0$. On the other hand, from the expression of $\Lambda$, one has

$$
\begin{aligned}
\Lambda+\sum_{j=1}^{d-1}\left(s_{d-j}-s_{d+j}\right) & =(k+t) x_{v_{2 d-1}}-k x_{v_{1}} \\
& =k \cdot \frac{\rho(G)}{\rho(G)+2}\left(x_{u_{2 d-1}}-x_{u_{1}}\right)+t x_{v_{2 d-1}} \\
& >0,
\end{aligned}
$$

which is a contradiction. It follows that $x_{u_{1}}>x_{u_{2 d-1}}$. Then $s_{1}>s_{2 d-1}$, so $\Lambda>0$. Therefore $s_{d-i}>s_{d+i}$ for $i \in[d-1]$. 
By the previous lemma and its proof, $s_{d-i}>s_{d+i}$ for $i \in[d-1]$, and $\Lambda>0$. So from (2.2), we have

Corollary 2.6. Let $G=E(n, \ell ; k, k+t)$, where $k \geq 0, t \geq 1, \ell=\frac{n+2-2 k-t}{2}=2 d$ and $d \geq 2$. Let $x$ be the $D(G)$-Perron vector and $s_{i}=x_{u_{i}}+x_{v_{i}}$ for $i \in[2 d-1]$. Then

$$
s_{d-i}-s_{d+i}>s_{d-i+1}-s_{d+i-1}
$$

for $i \in[d-1]$.

Lemma 2.7. Let $G=E(n, \ell ; k, k+t)$, where $k \geq 0, t \geq 1, \ell=\frac{n+2-2 k-t}{2}=2 d+1$, and $d \geq 2$. Let $x$ be the $D(G)$-Perron vector and $s_{i}=x_{u_{i}}+x_{v_{i}}$ for $i \in[2 d]$. Then $s_{d-i} \geq s_{d+i+1}$ for $0 \leq i \leq d-1$.

Proof. Let the vertices of $G$ be labelled as in Figure 2 (with $\ell=2 d+1$ ). Let $\Lambda=\sum_{j=d+1}^{2 d} s_{j}+(k+t) x_{v_{2 d}}-$ $\left(\sum_{j=1}^{d} s_{j}+k x_{v_{1}}\right)$.

Let $K=\rho(G)+\frac{\rho(G)}{\rho(G)+1}$. For $i=0, \ldots, d-1$, by Lemma 2.1, one has

$$
K\left(s_{d-i}-s_{d+i+1}\right)=2 \rho(G)\left(x_{u_{d-i}}-x_{u_{d+i+1}}\right),
$$

so, by the $D(G)$-eigenequations at $u_{d-i}$ and $u_{d+i+1}$, one has

$$
\begin{aligned}
K\left(s_{d-i}-s_{d+i+1}\right)= & -2(2 i+1) \sum_{j=1}^{d-i} s_{j}-2(2 i+1) k x_{v_{1}}-\sum_{j=0}^{i-1} 2(2 j+1) s_{d-j}+\sum_{j=0}^{i-1} 2(2 j+1) s_{d+j+1} \\
& +2(2 i+1) \sum_{j=d+i+1}^{2 d} s_{j}+2(2 i+1)(k+t) x_{v_{2 d}} .
\end{aligned}
$$

Thus, for $0 \leq i \leq d-1$, one has

$$
\begin{aligned}
& K\left(\left(s_{d-i}-s_{d+i+1}\right)-\left(s_{d-i+1}-s_{d+i}\right)\right) \\
= & 4\left(\sum_{j=d+i+1}^{2 d} s_{j}+(k+t) x_{v_{2 d}}-k x_{v_{1}}-\sum_{j=1}^{d-i} s_{j}\right) \\
= & 4 \Lambda+4 \sum_{j=0}^{i-1}\left(s_{d-j}-s_{d+j+1}\right) .
\end{aligned}
$$

It is easy to prove that $s_{d-i}-s_{d+i+1}$ and $\Lambda$ have the same sign by induction on $i$ for $0 \leq i \leq d-1$. If $i=0$, then it follows from (2.3). Suppose that $1 \leq i \leq d-1$. By the induction hypothesis, $s_{d-j}-s_{d+j+1}$ and $\Lambda$ have the same sign for $0 \leq j \leq i-1$. Thus $4 \sum_{j=0}^{i-1}\left(s_{d-j}-s_{d+j+1}\right)$ and $\Lambda$ have the same sign. Now from (2.3), $s_{d-i}-s_{d+i+1}$ and $\Lambda$ have the same sign. This shows that $s_{d-i}-s_{d+i+1}$ and $\Lambda$ have the same sign for $0 \leq i \leq d-1$, as desired.

Suppose that $x_{u_{1}} \leq x_{u_{2 d}}$. By Lemma 2.1, $s_{1} \leq s_{2 d}$. As $s_{d-i}-s_{d+i+1}$ and $\Lambda$ have the same sign for $0 \leq i \leq d-1$, one has $\Lambda \leq 0$. But, on the other hand, $\Lambda+\sum_{j=0}^{d-1}\left(s_{d-j}-s_{d+j+1}\right)=(k+t) x_{v_{2 d}}-k x_{v_{1}}=k \frac{\rho(G)}{\rho(G)+2}\left(x_{u_{2 d}}-x_{u_{1}}\right)+$ $t x_{v_{2 d}}>0$, a contradiction. It follows that $x_{u_{1}}>x_{u_{2 d}}$. Then $s_{1}>s_{2 d}$, so $\Lambda>0$. Therefore $s_{d-i}>s_{d+i+1}$ for $0 \leq i \leq d-1$.

Corollary 2.8. Let $G=E(n, \ell ; k, k+t)$, where $k \geq 0, t \geq 1, \ell=\frac{n+2-2 k-t}{2}=2 d+1$ and $d \geq 2$. Let $x$ be the $D(G)$-Perron vector and $s_{i}=x_{u_{i}}+x_{v_{i}}$ for $i \in[d-1]$. Then

$$
s_{d-i}-s_{d+i+1}>s_{d-i+1}-s_{d+i}
$$

for $0 \leq i \leq d-1$. 
Lemma 2.9. Suppose that $3 \leq \ell \leq\left\lfloor\frac{n}{2}\right\rfloor, k \geq 0, t \geq 2$ and $2 \ell+2 k+t-2=n$. Then $\rho(E(n, \ell ; k+1, k+t-1))>$ $\rho(E(n, \ell ; k, k+t))$.

Proof. If $\ell=3$, this is Corollary 3.2 of [4]. Suppose that $\ell \geq 4$. Let $G=E(n, \ell ; k, k+t)$. Let the vertices of $G$ be labelled as in Figure 2. Let $x$ be the $D(G)$-Perron vector. Let $G^{\prime}$ be the tree obtained from $G$ by deleting a leaf adjacent to $u_{\ell-1}$ that is different from $v_{\ell-1}$ and adding a leaf edge at $u_{1}$. Obviously, $G^{\prime} \cong E(n, \ell ; k+1, k+t-1)$.

Case 1. $\ell=2 d$.

Let $s_{i}=x_{u_{i}}+x_{v_{i}}$ for $i \in[2 d-1]$. Then

$$
\frac{1}{2}\left(\rho\left(G^{\prime}\right)-\rho(G)\right) \geq \frac{1}{2} x^{\top}\left(D\left(G^{\prime}\right)-D(G)\right) x=x_{v_{2 d-1}} F,
$$

where

$$
F=k(2 d-2)\left(x_{v_{2 d-1}}-x_{v_{1}}\right)+(t-1)(2 d-2) x_{v_{2 d-1}}+\sum_{i=1}^{d-1}(2 d-2 i)\left(s_{2 d-i}-s_{i}\right) .
$$

Suppose that $F \leq 0$.

For $i \in[d-1]$, by Corollary 2.6, $s_{i}-s_{2 d-i} \leq s_{1}-s_{2 d-1}$, and thus by Lemma 2.1,

$$
s_{i}-s_{2 d-i} \leq s_{1}-s_{2 d-1}=\frac{2 \rho(G)+2}{\rho(G)+2}\left(x_{u_{1}}-x_{u_{2 d-1}}\right)<2\left(x_{u_{1}}-x_{u_{2 d-1}}\right) .
$$

By Lemma 2.1,

$$
x_{v_{1}}-x_{v_{2 d-1}}=\frac{\rho(G)}{\rho(G)+2}\left(x_{u_{1}}-x_{u_{2 d-1}}\right)<x_{u_{1}}-x_{u_{2 d-1}} .
$$

Thus

$$
\begin{aligned}
\rho(G)\left(x_{u_{1}}-x_{u_{2 d-1}}\right)= & \sum_{v \in V(G)}\left(d_{G}\left(v, u_{1}\right)-d_{G}\left(v, u_{2 d-1}\right)\right) x_{v} \\
= & k(2-2 d) x_{v_{1}}+\sum_{i=1}^{d-1}(2 i-2 d) s_{i}+\sum_{i=1}^{d-1}(2 d-2 i) s_{2 d-i}+(k+t)(2 d-2) x_{v_{2 d-1}} \\
= & k(2 d-2)\left(x_{v_{2 d-1}}-x_{v_{1}}\right)+t(2 d-2) x_{v_{2 d-1}}+\sum_{i=1}^{d-1}(2 d-2 i)\left(s_{2 d-i}-s_{i}\right) \\
= & 2 F-(t-2)(2 d-2) x_{v_{2 d-1}}+\sum_{i=1}^{d-1}(2 d-2 i)\left(s_{i}-s_{2 d-i}\right) \\
& +k(2 d-2)\left(x_{v_{1}}-x_{v_{2 d-1}}\right) \\
\leq & \sum_{i=1}^{d-1}(2 d-2 i)\left(s_{i}-s_{2 d-i}\right)+k(2 d-2)\left(x_{v_{1}}-x_{v_{2 d-1}}\right) \\
< & 2 \sum_{i=1}^{d-1}(2 d-2 i)\left(x_{u_{1}}-x_{u_{2 d-1}}\right)+k(2 d-2)\left(x_{u_{1}}-x_{u_{2 d-1}}\right) \\
= & \left(2 d^{2}-2 d+2 k d-2 k\right)\left(x_{u_{1}}-x_{u_{2 d-1}}\right)
\end{aligned}
$$

implying

$$
\left(\rho(G)-\left(2 d^{2}-2 d+2 k d-2 k\right)\right)\left(x_{u_{1}}-x_{u_{2 d-1}}\right)<0 .
$$


For $i \in[2 d-1]$,

$$
\begin{aligned}
s_{G}\left(u_{i}\right) & =\sum_{v \in V(G)} d_{G}\left(v, u_{i}\right) \\
& =2 i^{2}-(4 d+t) i+4 d^{2}+2 k d+2 d t-1 \\
& =2\left(i-\left(d+\frac{t}{4}\right)\right)^{2}+2 d^{2}+d t+2 k d-\frac{t^{2}}{8}-1 .
\end{aligned}
$$

Suppose first that $2 \leq t \leq 4 d-4$. Then $2 \leq d+\frac{t}{4} \leq 2 d-1$. As a function of $i, s_{G}\left(u_{i}\right)$ achieves its minimum if $i=d+\frac{t}{4}$, so

$$
s(G) \geq 2 d^{2}+d t+2 k d-\frac{t^{2}}{8}-1 .
$$

Let $f(t)=2 d^{2}+d t+2 k d-\frac{t^{2}}{8}-1$. As $f(t)$ is strictly increasing for $2 \leq t \leq 4 d, f(t) \geq f(2)=2 d^{2}+2 d+2 k d-\frac{3}{2}$. Thus $s(G) \geq 2 d^{2}+2 d+2 k d-\frac{3}{2}$. Suppose next that $t \geq 4 d-3$. Then $d+\frac{t}{4}>2 d-1$. As $s_{G}\left(u_{i}\right)$ is strictly decreasing for $i \in[2 d-1], s_{G}\left(u_{i}\right)$ achieves its minimum if $i=2 d-1$, so

$$
\begin{aligned}
s(G) & \geq 4 d^{2}+2 k d-4 d+t+1 \\
& \geq 4 d^{2}+2 k d-2 \\
& >2 d^{2}+2 d+2 k d-\frac{3}{2} .
\end{aligned}
$$

Thus $s(G) \geq 2 d^{2}+2 d+2 k d-\frac{3}{2}$ in either case. Note that $\rho(G) \geq s(G)[19]$. Thus

$$
\rho(G) \geq 2 d^{2}+2 d+2 k d-\frac{3}{2}
$$

which together with (2.4), implies $x_{u_{1}}<x_{u_{2 d-1}}$. But by Lemma 2.5, $x_{u_{1}}>x_{u_{2 d-1}}$, a contradiction. Thus $F>0$, and then $\rho\left(G^{\prime}\right)>\rho(G)$.

Case 2. $\ell=2 d+1$.

Let $s_{i}=x_{u_{i}}+x_{v_{i}}$ for $i \in[2 d]$. Then

$$
\frac{1}{2}\left(\rho\left(G^{\prime}\right)-\rho(G)\right) \geq \frac{1}{2} x^{\top}\left(D\left(G^{\prime}\right)-D(G)\right) x=x_{v_{2 d}} L,
$$

where

$$
L=k(2 d-1)\left(x_{v_{2 d}}-x_{v_{1}}\right)+(t-1)(2 d-1) x_{v_{2 d}}+\sum_{i=1}^{d}(2 d-2 i+1)\left(s_{2 d-i+1}-s_{i}\right) .
$$

Suppose that $L \leq 0$.

For $i \in[d]$, by Corollary 2.8 and Lemma 2.1, one has

$$
s_{i}-s_{2 d-i+1}<2\left(x_{u_{1}}-x_{u_{2 d}}\right)
$$

and

$$
x_{v_{1}}-x_{v_{2 d}}<x_{u_{1}}-x_{u_{2 d}} .
$$


Thus

$$
\begin{aligned}
\rho(G)\left(x_{u_{1}}-x_{u_{2 d}}\right)= & \sum_{v \in V(G)}\left(d_{G}\left(v, u_{1}\right)-d_{G}\left(v, u_{2 d}\right)\right) x_{v} \\
= & k(1-2 d) x_{v_{1}}+\sum_{i=1}^{d}(2 i-(2 d+1)) s_{i}+\sum_{i=1}^{d}(2 d+1-2 i) s_{2 d-i+1} \\
& +(k+t)(2 d-1) x_{v_{2 d}} \\
= & k(2 d-1)\left(x_{v_{2 d}}-x_{v_{1}}\right)+t(2 d-1) x_{v_{2 d}}+\sum_{i=1}^{d}(2 d-2 i+1)\left(s_{2 d-i+1}-s_{i}\right) \\
= & 2 L-(t-2)(2 d-1) x_{v_{2 d}}+\sum_{i=1}^{d}(2 d-2 i+1)\left(s_{i}-s_{2 d-i+1}\right) \\
& +k(2 d-1)\left(x_{v_{1}}-x_{v_{2 d}}\right) \\
\leq & \sum_{i=1}^{d}(2 d-2 i+1)\left(s_{i}-s_{2 d-i+1}\right)+k(2 d-1)\left(x_{v_{1}}-x_{v_{2 d}}\right) \\
< & 2 \sum_{i=1}^{d}(2 d-2 i+1)\left(x_{u_{1}}-x_{u_{2 d}}\right)+k(2 d-1)\left(x_{u_{1}}-x_{u_{2 d}}\right) \\
= & \left(2 d^{2}+2 k d-k\right)\left(x_{u_{1}}-x_{u_{2 d}}\right),
\end{aligned}
$$

implying

$$
\left(\rho(G)-\left(2 d^{2}+2 k d-k\right)\right)\left(x_{u_{1}}-x_{u_{2 d}}\right)<0 .
$$

For $i \in[2 d]$,

$$
\begin{aligned}
s_{G}\left(u_{i}\right) & =\sum_{v \in V(G)} d_{G}\left(v, u_{i}\right) \\
& =2 i^{2}-(4 d+t+2) i+4 d^{2}+4 d+2 k d+k+2 d t+t \\
& =2\left(i-\left(d+\frac{t+2}{4}\right)\right)^{2}+2 d^{2}+2 d+2 k d+k+d t+\frac{t}{2}-\frac{t^{2}}{8}-\frac{1}{2} .
\end{aligned}
$$

Arguing as in Case $1, s(G) \geq 2 d^{2}+4 d+2 k d+k$, so $\rho(G) \geq 2 d^{2}+4 d+2 k d+k$. It follows from (2.5) that $x_{u_{1}}<x_{u_{2 d}}$. But by Lemma 2.7, one has $x_{u_{1}}>x_{u_{2 d}}$, a contradiction. Thus $L>0$, so $\rho\left(G^{\prime}\right)>\rho(G)$.

\section{MAin Results}

In this section, we determine the trees with maximum distance spectral radius among all series-reduced trees with fixed order and maximum degree and among all series-reduced trees with fixed order and number of leaves, respectively.

For $3 \leq \Delta \leq n-1$, let $\mathcal{S R}(n, \Delta)$ be the set of series-reduced trees of order $n$ with the maximum degree $\Delta$. Obviously, $\mathcal{S R}(n, n-1)$ contains only the star $S_{n}$ of order $n, \mathcal{S R}(n, n-2)=\emptyset, \mathcal{S R}(n, n-3)$ contains only the double star with degrees $n-3$ and 3 for its centers, and $\mathcal{S R}(n, n-4)$ contains only the double star with degrees $n-4$ and 4 for its centers.

Theorem 3.1. Let $T \in \mathcal{S R}(n, \Delta)$, where $3 \leq \Delta \leq n-5$. Then

$$
\rho(T) \leq \rho\left(E\left(n,\left\lceil\frac{n-\Delta}{2}\right\rceil+1 ;\left\lfloor\frac{n-\Delta}{2}\right\rfloor-\left\lceil\frac{n-\Delta}{2}\right\rceil+2, \Delta-2\right)\right)
$$


with equality if and only if $T \cong E\left(n,\left\lceil\frac{n-\Delta}{2}\right\rceil+1 ;\left\lfloor\frac{n-\Delta}{2}\right\rfloor-\left\lceil\frac{n-\Delta}{2}\right\rceil+2, \Delta-2\right)$.

Proof. Let $T$ be a tree in $\mathcal{S R}(n, \Delta)$ that maximizes the distance spectral radius. By [5], the maximum distance spectral radius of a tree with given degree sequence is uniquely attained by a caterpillar. So $T$ is a caterpillar.

If $\Delta=3$, then $n$ is even, $n-\Delta$ is odd and $T \cong E\left(n, \frac{n}{2} ; 1,1\right)$.

Suppose in the following that $4 \leq \Delta \leq n-5$.

Let $d$ be the diameter of $T$ and let $u_{0} \ldots u_{d}$ be a path of length $d$ in $T$. Let $I(T)$ be the set of internal vertices of $T$, i.e., $I(T)=\left\{u_{i}: 1 \leq i \leq d-1\right\}$. Let $u$ be a vertex of degree $\Delta$. Evidently, $u \in I(T)$. For $i \in[d-1]$, let $v_{i}$ be a leaf at $u_{i}$ in $T$.

Case 1. $\Delta=4$.

Suppose that $\delta_{T}\left(u_{1}\right) \neq 4$ and $\delta_{T}\left(u_{d-1}\right) \neq 4$. As $T$ is a series-reduced tree, $\delta_{T}\left(u_{1}\right)=\delta_{T}\left(u_{d-1}\right)=3$. Then $u=u_{i_{0}}$ for some $1<i_{0}<d-1$. Let $T_{1}$ be the component of $T-u$ containing $u_{0}$ and $T_{2}$ the component of $T-u$ containing $u_{d}$. Let $T^{\prime}=T-u v_{i_{0}}+u_{1} v_{i_{0}}$ and $T^{\prime \prime}=T-u v_{i_{0}}+u_{d-1} v_{i_{0}}$. Note that $T^{\prime}, T^{\prime \prime} \in \mathcal{S R}(n, \Delta)$. By Lemma 2.3, $\rho\left(T^{\prime}\right)>\rho(T)$ or $\rho\left(T^{\prime \prime}\right)>\rho(T)$, a contradiction. So $u$ may be chosen to be $u_{d-1}$.

Suppose that there are three vertices of degree 4 . Recall that $\delta_{T}\left(u_{d-1}\right)=4$. Choose a vertex $u_{i}$ of degree 4 with the smallest $i$. There is also a vertex $u_{j}$ of degree 4 with $1 \leq i<j<d-1$. Let $F_{1}$ and $F_{2}$ be the components of $T-u_{j}$ containing $u_{0}$ and $u_{d}$, respectively. Assume that $\sigma_{T}\left(F_{1}\right) \leq \sigma_{T}\left(F_{2}\right)$ (otherwise change the role of $u_{i}$ and $\left.u_{d-1}\right)$. Let $T^{\prime}=T-u_{j} v_{j}+u_{i} v_{j}$. By Lemma 2.3, $\rho\left(T^{\prime}\right)>\rho(T)$. Note that the maximum degree of $T^{\prime}$ is 5 . Let $F_{1}^{\prime}$ and $F_{2}^{\prime}$ be the components of $T^{\prime}-u_{i}$ containing $u_{0}$ and $u_{d}$, respectively. Let $T^{*}=T^{\prime}-\left\{u_{i} v_{i}, u_{i} v_{j}\right\}+\left\{u_{0} v_{i}, u_{0} v_{j}\right\}$ and $T^{* *}=T^{\prime}-\left\{u_{i} v_{i}, u_{i} v_{j}\right\}+\left\{u_{d} v_{i}, u_{d} v_{j}\right\}$. Note that $T^{*}, T^{* *} \in \mathcal{S R}(n, 4)$. By Lemma $2.3, \rho\left(T^{*}\right)>\rho\left(T^{\prime}\right)$ or $\rho\left(T^{* *}\right)>\rho\left(T^{\prime}\right)$, so $\rho\left(T^{*}\right)>\rho(T)$ or $\rho\left(T^{* *}\right)>\rho(T)$, a contradiction. Hence, there are at most two vertices of degree 4 in $T$, and one is $u_{d-1}$.

If $u_{d-1}$ is the only vertex of degree 4 in $T$, then $\delta_{T}\left(u_{i}\right)=3$ for $i \in[d-2]$ and $n-\Delta=2 d-3$, so $T \cong E\left(n, \frac{n-1}{2} ; 1,2\right)$.

Suppose that there are exactly two vertices of degree 4 in $T$. By Theorem 6 of [5], the other vertex of degree 4 must be $u_{1}$ or $u_{d-2}$. Then $n-4=2 d-2$.

Suppose that $\delta_{T}\left(u_{1}\right) \neq 4$. Then $\delta_{T}\left(u_{d-2}\right)=4$. Let $T_{1}$ be the component of $T-u_{d-2}$ containing $u_{0}$ and $T_{2}$ the component of $T-u_{d-2}$ containing $u_{d}$. Suppose that $\sigma_{T}\left(T_{1}\right) \leq \sigma_{T}\left(T_{2}\right)$. Let $T^{\prime}=T-u_{d-2} v_{d-2}+u_{1} v_{d-2}$. Obviously, $T^{\prime} \cong E\left(n, \frac{n-2}{2} ; 2,2\right)$. By Lemma $2.3, \rho\left(T^{\prime}\right)>\rho(T)$, a contradiction. It follows that $\sigma_{T}\left(T_{1}\right)>$ $\sigma_{T}\left(T_{2}\right)$. Let $T^{\prime}=T-u_{d-2} v_{d-2}+u_{d-1} v_{d-2}$. Obviously, $\delta_{T^{\prime}}\left(u_{d-1}\right)=5, \delta_{T^{\prime}}\left(u_{d-2}\right)=3$ and $\delta_{T^{\prime}}(w)=\delta_{T}(w)$ for $w \in V\left(T^{\prime}\right) \backslash\left\{u_{d-1}, u_{d-2}\right\}$. Let $T^{\prime \prime}=T^{\prime}-u_{d-1} v_{d-2}+u_{1} v_{d-2}$. Note that $T^{\prime} \cong E\left(n, \frac{n-2}{2} ; 1,3\right)$ and $T^{\prime \prime} \cong$ $E\left(n, \frac{n-2}{2} ; 2,2\right)$. By Lemmas 2.9 and $2.3, \rho\left(T^{\prime \prime}\right)>\rho\left(T^{\prime}\right)>\rho(T)$, also a contradiction. Hence $\delta_{T}\left(u_{1}\right)=4$. That is, $T \cong E\left(n, \frac{n-2}{2} ; 2,2\right)$.

Case 2. $\Delta \geq 5$.

Suppose that there is a vertex, say $w$, in $T$ different from $u$ with degree larger than 4 . Then $w=u_{j_{0}}$ for some $j_{0} \in[d-1]$. Let $r=\delta_{T}(w)$. Then $5 \leq r \leq \Delta$. Let $T_{1}$ be the component of $T-w$ containing $u_{0}$ and $T_{2}$ the component of $T-w$ containing $u_{d}$. Assume that $\sigma_{T}\left(T_{1}\right) \geq \sigma_{T}\left(T_{2}\right)$. Let $T^{\prime}=T-\left\{w v_{j_{0}}, w v_{j_{0}^{\prime}}\right\}+\left\{u_{d} v_{j_{0}}, u_{d} v_{j_{0}^{\prime}}\right\}$, where $v_{j_{0}}^{\prime}$ is a leaf at $u_{j_{0}}$ in $T$ different from $v_{j_{0}}$. Evidently, $\delta_{T^{\prime}}(w)=r-2, \delta_{T^{\prime}}\left(u_{d}\right)=3$, and $\delta_{T^{\prime}}(z)=\delta_{T}(z)$ for $z \in V(T) \backslash\left\{w, u_{d}\right\}$. So $T^{\prime} \in \mathcal{S R}(n, \Delta)$. By Lemma 2.3, $\rho\left(T^{\prime}\right)>\rho(T)$, a contradiction. So the degree of any internal vertex different from $u$ is 3 or 4 in $T$.

Suppose that $\delta_{T}\left(u_{1}\right) \neq \Delta$ and $\delta_{T}\left(u_{d-1}\right) \neq \Delta$. Then $\delta_{T}\left(u_{d-1}\right)=3,4$. Let $T_{1}$ be the component of $T-u$ containing $u_{0}$ and $T_{2}$ the component of $T-u$ containing $u_{d}$. Let $N$ be the set of some $\Delta-\delta_{T}\left(u_{d-1}\right)$ leaves at $u$ in $T$. Assume that $\sigma_{T}\left(T_{1}\right) \geq \sigma_{T}\left(T_{2}\right)$. Let $T^{\prime}=T-\{u z: z \in N\}+\left\{u_{d-1} z: z \in N\right\}$. Obviously, $T^{\prime} \in \mathcal{S R}(n, \Delta)$. By Lemma 2.3, $\rho\left(T^{\prime}\right)>\rho(T)$, a contradiction. So $u$ may be chosen to be $u_{d-1}$.

Suppose that there are three vertices, say $u_{i}, u_{j}, u_{\ell}$, of degree 4 in $T$, where $1 \leq i<j<\ell \leq d-2$. Let $T^{\prime}=T-u_{j} v_{j}+u_{1} v_{j}$ and $T^{\prime \prime}=T-u_{j} v_{j}+u_{d-2} v_{j}$. Then $T^{\prime}, T^{\prime \prime} \in \mathcal{S R}(n, \Delta)$. By Lemma $2.3, \rho\left(T^{\prime}\right)>\rho(T)$ or $\rho\left(T^{\prime \prime}\right)>\rho(T)$, a contradiction. So there are at most two vertices of degree 4 in $T$. 
Suppose that there are exactly two vertices of degree 4 in $T$. Then $n-\Delta=2 d-1$ and $\delta_{T}\left(u_{i}\right)=\delta_{T}\left(u_{j}\right)=4$ for some $i$ and $j$ with $1 \leq i<j \leq d-2$. Suppose that $j<d-2$. Let $T^{\prime}=T-u_{j} v_{j}+u_{1} v_{j}$ and $T^{\prime \prime}=T-u_{j} v_{j}+u_{d-2} v_{j}$. By Lemma 2.3, $\rho\left(T^{\prime}\right)>\rho(T)$ or $\rho\left(T^{\prime \prime}\right)>\rho(T)$, a contradiction. So $j=d-2$. By similar argument $i \geq 2$ is impossible, so $i=1$.

Let $T_{1}$ and $T_{2}$ be the components of $T-u_{d-2}$ containing $u_{1}$ and $u_{d}$, respectively. Suppose first that $\sigma_{T}\left(T_{1}\right) \leq \sigma_{T}\left(T_{2}\right)$. Let $T^{\prime}=T-u_{d-2} v_{d-2}+u_{1} v_{d-2}$. Then $\delta_{T^{\prime}}\left(u_{d-2}\right)=3, \delta_{T^{\prime}}\left(u_{1}\right)=5$ and $\delta_{T^{\prime}}(z)=\delta_{T}(z)$ for $z \in V\left(T^{\prime}\right) \backslash\left\{u_{1}, u_{d-2}\right\}$. So $T^{\prime} \in \mathcal{S R}(n, \Delta)$. By Lemma 2.3, $\rho\left(T^{\prime}\right)>\rho(T)$, a contradiction. It follows that $\sigma_{T}\left(T_{1}\right)>\sigma_{T}\left(T_{2}\right)$. Let $T^{\prime}=T-u_{d-2} v_{d-2}+u_{d-1} v_{d-2}$. Then $\delta_{T^{\prime}}\left(u_{d-2}\right)=3, \delta_{T^{\prime}}\left(u_{d-1}\right)=\Delta+1$ and $\delta_{T^{\prime}}(z)=\delta_{T}(z)$ for $z \in V\left(T^{\prime}\right) \backslash\left\{u_{d-1}, u_{d-2}\right\}$. That is, $T^{\prime} \in \mathcal{S R}(n, \Delta+1)$. By Lemma 2.3, $\rho\left(T^{\prime}\right)>\rho(T)$. Let $T^{\prime \prime}=T^{\prime}-u_{1} v_{1}+u_{0} v_{1}$. Then $\delta_{T^{\prime \prime}}\left(u_{0}\right)=2, \delta_{T^{\prime \prime}}\left(u_{i}\right)=3$ for $i \in[d-2]$ and $\delta_{T^{\prime \prime}}\left(u_{d-1}\right)=\Delta+1$. By Lemma $2.2, \rho\left(T^{\prime \prime}\right)>\rho\left(T^{\prime}\right)$. Let $\widehat{T}=T^{\prime \prime}-u_{d-1} v_{d-2}+u_{0} v_{d-2}$. Then $\delta_{\widehat{T}}\left(u_{i}\right)=3$ for $0 \leq i \leq d-2$ and $\delta_{\widehat{T}}\left(u_{d-1}\right)=\Delta$, so $\widehat{T} \in \mathcal{S R}(n, \Delta)$. Note that $T^{\prime \prime} \cong E\left(n, \frac{n-\Delta+3}{2} ; 0, \Delta-1\right)$, and $\widehat{T} \cong E\left(n, \frac{n-\Delta+3}{2} ; 1, \Delta-2\right)$. By Lemma $2.9, \rho(\widehat{T})>\rho\left(T^{\prime \prime}\right)$. So $\rho(\widehat{T})>\rho(T)$, a contradiction. Hence, there are at most one vertex of degree 4 in $T$.

If there is no vertex of degree 4 , then $n-\Delta=2 d-3$ and $T \cong E\left(n, \frac{n-\Delta+3}{2} ; 1, \Delta-2\right)$.

Suppose that there is exactly one vertex with degree 4 in $T$, and by (K. Dadedzi, V. Razanajatovo Misanantenaina and S. Wagner [5] Thm. 6), this vertex is $u_{1}$ or $u_{d-2}$. Note that $n-\Delta=2 d-2$. If $\delta_{T}\left(u_{1}\right) \neq 4$, then $\delta_{T}\left(u_{d-2}\right)=4$, so by similar argument as in Case 1 by using Lemmas 2.3 and 2.9, a contradiction occurs. So $\delta_{T}\left(u_{1}\right)=4$ and $T \cong E\left(n, \frac{n-\Delta+2}{2} ; 2, \Delta-2\right)$.

Let $T$ be a series-reduced trees of order $n$ with $p$ leaves. Denote by $n_{i}$ the number of vertices of degree $i$ in $T$, where $i=1, \ldots, \Delta$ with $\Delta$ being the maximum degree of $T$. Evidently, $n_{2}=0$. Then $\sum_{i=1}^{\Delta} n_{i}=n$ and $\sum_{i=1}^{\Delta} i n_{i}=2(n-1)$. So $2 n_{1}=n+2+\sum_{i=4}^{\Delta}(i-3) n_{i}$, and $n_{1} \geq \frac{n+2}{2}$.

Let $\mathbb{S R}(n, p)$ be the set of series-reduced trees of order $n$ with $p$ leaves, where $\left\lceil\frac{n+2}{2}\right\rceil \leq p \leq n-1$. It is evident that $\mathbb{S R}(n, n-1)=\left\{S_{n}\right\}$ for $n \geq 4$.

Theorem 3.2. Let $T \in \mathbb{S R}(n, p)$, where $\left\lceil\frac{n+2}{2}\right\rceil \leq p \leq n-2$. Then

$$
\rho(T) \leq \rho\left(E\left(n, n-p+1 ;\left\lfloor\frac{2 p-n}{2}\right\rfloor,\left\lceil\frac{2 p-n}{2}\right\rceil\right)\right)
$$

with equality if and only if $T \cong E\left(n, n-p+1 ;\left\lfloor\frac{2 p-n}{2}\right\rfloor,\left\lceil\frac{2 p-n}{2}\right\rceil\right)$.

Proof. Let $T$ be a tree in $\mathbb{S R}(n, p)$ that maximizes the distance spectral radius.

First, we show that $T$ is a caterpillar. Suppose that this is not true. Let $F$ be the tree obtained from $T$ by deleting all leaves. Denote by $r$ the maximum degree of $F$. Then $r \geq 3$. Suppose that $r \geq 4$, say $\delta_{F}(w)=r \geq 4$ for some $w \in V(F)$. Let $w_{1}, \ldots, w_{r}$ be the neighbors of $w$ in $F$. Obviously, $\left\{w_{1}, \ldots, w_{r}\right\} \subseteq N_{T}(w)$. For $i=1,2,3$, let $T_{i}$ be the component of $T-w$ containing $w_{i}$. Assume that $\sigma_{T}\left(T_{1}\right) \geq \sigma_{T}\left(T_{2}\right)$. Let $T^{\prime}=T-w w_{3}+w_{2} w_{3}$. Then $T^{\prime} \in \mathbb{S R}(n, p)$. By Lemma 2.3, $\rho\left(T^{\prime}\right)>\rho(T)$, a contradiction. It thus follows that $r=3$. So there is a vertex $w$ with degree 3 in $F$ such that there are two paths, say $P$ and $Q$, at $w$ in $F$ such that any internal vertex of $F$ on these two paths except $w$ (if any exists) has degree two. Let $w_{1}, w_{2}$ and $w_{3}$ be the neighbors of $w$ in $F$, where $w_{1} \in V(P)$ and $w_{2} \in V(Q)$. Then $\delta_{T}\left(w_{i}\right) \geq 3$ for $i=1,2,3$. By similar argument as above, $\delta_{T}(w)=3$, implying that $w$ is not adjacent to any leaf in $T$. By the choice of $w$, there is a leaf $z_{i}$ adjacent to $w_{i}$ in $T$ for each $i=1,2$. Let $T^{\prime}=T-\left\{w w_{3}, w_{1} z_{1}\right\}+\left\{w_{1} w_{3}, w z_{1}\right\}$ and $T^{*}=T-\left\{w w_{3}, w_{2} z_{2}\right\}+\left\{w_{2} w_{3}, w z_{2}\right\}$. Note that $T^{\prime}, T^{*} \in \mathbb{S R}(n, p)$. By Lemma 2.4, $\max \left\{\rho\left(T^{\prime}\right), \rho\left(T^{*}\right)\right\}>\rho(T)$, also a contradiction. This shows that $T$ is a caterpillar.

If there is no vertex of degree larger than or equal to 4 in $T$, then it is trivial that $T \cong E\left(n, \frac{n}{2} ; 1,1\right)$, i.e., $T \cong E(n, n-p+1 ; 1,1)$ as in this case $p=\frac{n}{2}+1$.

Suppose that $\delta_{T}(u) \geq 4$ for some $u \in V(T)$. Let $\ell$ be the diameter of $T$ and $P=u_{0} \ldots u_{\ell}$ be a path of length $\ell$ in $T$. As $T$ is a caterpillar, $u$ lies on the path $P$. Suppose that $u \notin\left\{u_{1}, u_{\ell-1}\right\}$. Then $\ell \geq 4$. Let $T_{1}$ be the component of $T-u$ containing $u_{1}$ and $T_{2}$ the component of $T-u$ containing $u_{\ell-1}$. Assume that 
$\sigma_{T}\left(T_{1}\right) \geq \sigma_{T}\left(T_{2}\right)$. Let $z$ be a leaf adjacent to $u$. Let $T^{\prime}=T-u z+v_{\ell-1} z$. Obviously, $T^{\prime} \in \mathbb{S R}(n, p)$. By Lemma 2.3 , $\rho\left(T^{\prime}\right)>\rho(T)$, a contradiction. Therefore $u \in\left\{u_{1}, u_{\ell-1}\right\}$, and $T \cong E(n, \ell ; k, k+t)$ for some $k, t$ with $k \geq 1$, $t \geq 0$, and $\frac{n+2-2 k-t}{2}=\ell=n-p+1$. So $T \cong E(n, n-p+1 ; k, k+t)$ for some $k, t$ with $k \geq 1$ and $t \geq 0$, where $2 p-2 k-t=n$. Suppose that $t \geq 2$. Let $\widehat{T}=E(n, n-p+1 ; k+1, k+t-1)$. Obviously, $\widehat{T}$ is a series-reduced tree of order $n$ with $p$ leaves. By Lemma $2.9, \rho(\widehat{T})=\rho(E(n, n-p+1 ; k+1, k+t-1))>\rho(E(n, n-p+1 ; k, k+t))=\rho(T)$, a contradiction. So $t=0,1$, i.e.,

$$
T \cong E\left(n, n-p+1 ;\left\lfloor\frac{2 p-n}{2}\right\rfloor,\left\lceil\frac{2 p-n}{2}\right\rceil\right),
$$

completing the proof.

For a graph $G$, let $\Delta(G)$ be the maximum degree of $G$. Let $T$ be a series-reduced tree of order $n$ with maximum distance spectral radius, where $n \geq 4$. Then (H. Lin and B. Zhou [15] Lem. 4.1) states that $\Delta(T) \leq 4$, and there are at most two vertices of degree 4 in $T$. The argument for the second part there used the fact that $T$ is a caterpillar without proof. A proof is given below. Suppose that $T$ is not a caterpillar. Let $F$ be the tree obtained from $T$ by deleting all leaves. Then $\Delta(F)=3$, Suppose that $\Delta(F)=4$, say $\delta_{F}(u)=4$ with $u \in V(F)$. Assume that $N_{F}(u)=\left\{u_{1}, \ldots, u_{4}\right\}$. Evidently, $u_{1}, \ldots, u_{4}$ are also the neighbors of $u$ in $T$. For $i=1,2$, let $T_{i}$ be the component of $T-u$ containing $u_{i}$. Assume that $\sigma_{T}\left(T_{1}\right) \geq \sigma_{T}\left(T_{2}\right)$. Let $T^{\prime}=T-u u_{4}+u_{2} u_{4}$. Note that $\delta_{T^{\prime}}(u)=3, \delta_{T^{\prime}}\left(u_{2}\right)=4,5$ and $\delta_{T^{\prime}}(v)=\delta_{T}(v)$ for $v \in V(T) \backslash\left\{u, u_{2}\right\}$. Then $T^{\prime}$ is a series-reduced tree of order $n$. By Lemma 2.3, $\rho\left(T^{\prime}\right)>\rho(T)$, which is a contradiction. It follows that $\Delta(F)=3$. Then there is a vertex $u$ with degree 3 in $F$ such that there are two paths, say $P$ and $Q$, at $u$ in $F$ such that any internal vertex of $F$ except $u$ (if any exists) has degree two. Let $u_{1}, u_{2}$ and $u_{3}$ be the neighbors of $u$ in $F$, where $u_{1} \in V(P)$ and $u_{2} \in V(Q)$. By the choice of $F$, there is a leaf $v_{i}$ that is adjacent to $u_{i}$ in $T$, where $i=1,2$. Let $G_{1}=T-\left\{u u_{3}, u_{1} v_{1}\right\}+\left\{u_{1} u_{3}, u v_{1}\right\}$ and $G_{2}=T-\left\{u u_{3}, u_{2} v_{2}\right\}+\left\{u_{2} u_{3}, u v_{2}\right\}$. As $T$ is a series-reduced tree, $G_{1}$ and $G_{2}$ are also series-reduced trees and $\delta_{T}\left(u_{3}\right) \geq 3$. Then by Lemma $2.4, \max \left\{\rho\left(G_{1}\right), \rho\left(G_{2}\right)\right\}>\rho(T)$, a contradiction. Thus $T$ is a caterpillar. So, we have the following finer version of (H. Lin and B. Zhou [15] Lem. 4.1): 'Let $T$ be a series-reduced tree of order $n$ with maximum distance spectral radius, where $n \geq 4$. Then $\Delta(T) \leq 4, T$ is a caterpillar, and there are at most two vertices of degree 4 in $T$ '. With this, (H. Lin and B. Zhou [15] Thms. 4.1 and 4.2) follow by a simpler proof.

\section{Concluding Remarks}

For a caterpillar $T$ of diameter $\ell \geq 2$ with a path $v_{0} \ldots v_{\ell}$ of length $\ell$, let $s_{i}=\delta_{T}\left(v_{i}\right)-2$ for $i \in[\ell-1]$. Denote such a tree $T$ by $D\left(s_{1}, \ldots, s_{\ell-1}\right)$. In the following Table 1 , we list the maximum degree $\Delta$, number of leaves $p$, the domination number $\gamma$, diameter $d$ and the distance spectral radius $\rho$ of all 11-vertex series-reduced trees $T$ except the star, where $D(1,2,1)^{+}$is the tree obtained from $D(1,2,1)$ by adding two leaf edges at a leaf incident to $v_{2}$, and $D(1,1,1)^{+}$is the tree obtained from $D(1,1,1)$ by adding three leaf edges at a leaf incident to $v_{2}$. This demonstrates the validity of Theorems 3.1 and 3.2 . Note that $D(1,2,1)^{+}$and $D(1,1,1)^{+}$are not caterpillars. Though the extremal trees in Theorems 3.1 and 3.2 are caterpillars, we find from Table 1 that a tree that maximizes the distance spectral radius over all series-reduced trees with fixed order and domination number or diameter may be not a caterpillar, as $D(1,1,1)^{+}$is such a tree when $\gamma=3$ or when $d=4$.

It is a challenge to explore the connection between spectral properties and structural properties of graphs. In this paper, the spectral properties of a graph are studied through its distance matrix. The structural properties are considered through maximum degree and number of leaves. The trees with maximum distance spectral radius are identified over all series-reduced trees with given order and maximum degree, and over all seriesreduced caterpillars with given order and number of leaves, respectively. Generally, the tree with maximum distance spectral radius over all trees with given order and some parameter like maximum degree, number of leaves, matching number, and domination number may possess vertices of degree two. So, to determine the series-reduced trees with maximum distance spectral radius over all series-reduced trees with given order and 
TABLE 1. Values of $\Delta, p, \gamma, d$ and $\rho$ of 11 -vertex series-reduced trees $T$ except $S_{11}$.

\begin{tabular}{llllll}
\hline \hline$T$ & $\Delta$ & $p$ & $\gamma$ & $d$ & $\rho$ \\
\hline$D(1,6)$ & 8 & 9 & 2 & 3 & 21.3167 \\
$D(2,5)$ & 7 & 9 & 2 & 3 & 22.0147 \\
$D(3,4)$ & 6 & 9 & 2 & 3 & 22.3515 \\
$D(1,4,1)$ & 6 & 8 & 3 & 4 & 24.0353 \\
$D(1,1,4)$ & 6 & 8 & 3 & 4 & 25.1144 \\
$D(1,3,2)$ & 5 & 8 & 3 & 4 & 24.7391 \\
$D(1,2,3)$ & 5 & 8 & 3 & 4 & 25.0919 \\
$D(2,1,3)$ & 5 & 8 & 3 & 4 & 25.7805 \\
$D(2,2,2)$ & 4 & 8 & 3 & 4 & 25.4386 \\
$D(1,1,1)^{+}$ & 4 & 7 & 3 & 4 & 27.4185 \\
$D(1,2,1)^{+}$ & 4 & 7 & 4 & 4 & 26.7082 \\
$D(1,1,2,1)$ & 4 & 7 & 4 & 5 & 27.3587 \\
$D(1,1,1,2)$ & 4 & 7 & 4 & 5 & 28.5063 \\
\hline
\end{tabular}

some other parameter needs more deliberate analysis. As mentioned above, though the extremal trees in this paper are all caterpillars, there are also other classes of series-reduced trees for which the extremal trees are not caterpillars. It would be interesting to determine those series-reduced trees with maximum distance spectral radius over all series-reduced trees with given order and some other parameter, like domination number and diameter.

Recall that the distance spread of a connected graph is defined to be the difference between the largest and the smallest eigenvalues of its distance matrix [13]. A somewhat different research direction is to study the extremal problems for the distance spread of series-reduced trees.

Acknowledgements. The authors would like to thank the editor and referees for their kind comments and suggestions. This work was supported by National Natural Science Foundation of China (No. 11671156).

\section{REFERENCES}

[1] M. Aouchiche and P. Hansen, Distance spectra of graphs: a survey. Linear Algebra Appl. 458 (2014) 301-386.

[2] A.T. Balaban, D. Ciubotariu and M. Medeleanu, Topological indices and real number vertex invariants based on graph eigenvalues or eigenvectors. J. Chem. Inf. Comput. Sci. 31 (1991) 517-523.

[3] F. Bergeron, P. Leroux and G. Labelle, Combinatorial Species and Tree-like Structures. Cambridge University Press, Cambridge (1998).

[4] S.S. Bose, M. Nath and S. Paul, Distance spectral radius of graphs with $r$ pendent vertices. Linear Algebra Appl. 435 (2011) $2828-2836$.

[5] K. Dadedzi, V. Razanajatovo Misanantenaina and S. Wagner, On the distance spectral radius of trees with given degree sequence. Discuss. Math. Graph Theory 40 (2020) 495-524.

[6] Z. Du, A. Ilić and L. Feng, Further results on the distance spectral radius of graphs. Linear Multilinear Algebra 61 (2013) $1287-1301$.

[7] T.H. Foregger, Identities related to permanents of doubly stochastic matrices and series reduces trees. Linear Multilinear Algebra 7 (1979) 37-41.

[8] R.L. Graham and H.O. Pollak, On the addressing problem for loop switching. Bell Syst. Tech. J. 50 (1971) $2495-2519$.

[9] I. Gutman and M. Medeleanu, On the structure-dependence of the largest eigenvalue of the distance matrix of an alkane. Indian J. Chem. A $\mathbf{3 7}$ (1998) 569-573.

[10] J. Haslegrave, Extremal results on average subtree density of series-reduced trees. J. Combin. Theory Ser. B. 107 (2014) $26-41$.

[11] F. Harary and G. Prins, The number of homeomorphically irreducible trees, and other species. Acta Math. 101 (1959) 141-162.

[12] A. Ilić, Distance spectral radius of trees with given matching number. Discrete Appl. Math. 158 (2010) 1799-1806.

[13] Y. Liang and B. Zhou, On the distance spread of cacti and bicyclic graphs. Discrete Appl. Math. 206 (2016) $195-202$.

[14] H. Lin and B. Zhou, The distance spectral radius of graphs with given number of odd vertices. Electron. J. Linear Algebra 31 (2016) 286-305. 
[15] H. Lin and B. Zhou, The distance spectral radius of trees. Linear Multilinear Algebra 67 (2019) 370-390.

[16] H. Lin and B. Zhou, Distance spectral radius of trees with given number of segments. Linear Algebra Appl. 600 (2020) 40-59.

[17] W. Lin, Y. Zhang, Q. Chen, J. Chen, C. Ma and J. Chen, Ordering trees by their distance spectral radii. Discrete Appl. Math. 203 (2016) 106-110.

[18] Z. Luo and B. Zhou, On distance spectral radius of trees and fixed maximum degree. Filomat 29 (2015) $2021-2026$.

[19] H. Minc, Nonnegative Martices. John Wiley \& Sons, New York (1988).

[20] M. Nath and S. Paul, On the distance spectral radius of trees. Linear Multilinear Algebra 61 (2013) 847-855.

[21] W. Ning, L. Ouyang and M. Lu, Distance spectral radius of trees with fixed number of pendent vertices. Linear Algebra Appl. 439 (2013) 2240-2249.

[22] Z. Peng and B. Zhou, Minimum status of trees with given parameters. RAIRO-Oper. Res. (2020) DOI: https://doi.org/10.1051/ro/2020015.

[23] S.N. Ruzieh and D.L. Powers, The distance spectrum of the path $P_{n}$ and the first distance eigenvector of connected graphs. Linear Multilinear Algebra 28 (1990) 75-81.

[24] D. Stevanović and A. Ilić, Distance spectral radius of trees with fixed maximum degree. Electron. J. Linear Algebra 20 (2010) $168-179$.

[25] Y. Wang and B. Zhou, On distance spectral radius of graphs. Linear Algebra Appl. 438 (2013) 3490-3503.

[26] Y. Wang, R. Xing, B. Zhou and F. Dong, A note on distance spectral radius of trees. Spec. Matrices 5 (2017) $296-300$.

[27] R. Xing, B. Zhou and F. Dong, The effect of a graft transformation on distance spectral radius. Linear Algebra Appl. 457 (2014) 261-275.

[28] G. Yu, S. Guo and M. Zhai, Distance spectral radius of a tree with given diameter. Ars Combin. 134 (2017) 351-362.

[29] B. Zhou and N. Trinajstić, On the largest eigenvalue of the distance matrix of a connected graph. Chem. Phys. Lett. 447 (2007) 384-387. 